\title{
Control of Uniformity of Plasma-Surface Modification Inside of Small-Diameter Polyethylene Tubing Using Microplasma Diagnostics
}

Jason L. Lauer, Member, IEEE, J. Leon Shohet, Fellow, IEEE, Ralph M. Albrecht, Stephane Esnault, James S. Malter, Ulrich H. von Andrian, and Stephen B. Shohet

\begin{abstract}
A hollow-cathode microplasma was used to modify the lumenal surface of small-diameter polyethylene (PE). We make use of two microplasma diagnostics to monitor the plasma properties during the treatment process. A microwave cavity was used to measure the density of the microplasma. Emitted light from the microplasma was fed into a monochromator at various positions along the PE tube to assess uniformity of the microplasma. Effectiveness of plasma treatments were evaluated using the capillary-rise method at various positions along the tubing. We show a correlation between the properties of the inner surface of the PE tubing and the light emitted from the plasma. A Poly(ethylene oxide) (PEO) surfactant was immobilized to the lumenal surface of the PE tubing using the microplasma discharge. An in vitro blood-circulation loop was constructed to test the hematocompatibility of the PE tubes. After blood exposure, scanning electron microscope images were taken to assess the density of adhering platelets along the length of the tubes. The plasma-treated tubing showed fewer blood adherents than the untreated tubing. By suitably controlling the pressure drop along the tube, the uniformity of the microplasma treatment along the tubing can be optimized.
\end{abstract}

Index Terms-Argon plasma immobilization, biomaterials, blood, capillary rise method, hematocompatibility, hollow cathode discharges, microplasma diagnostics, microplasma surface modification, microwave cavity, polyethylene oxide (PEO), small-diameter polymer tubing, vascular grafts.

\section{INTRODUCTION}

$\mathbf{T}$ HE REALIZATION of small-scale biomedical devices will be closely related to the nonfouling/biocompatible properties of the exposed surfaces and the uniformity of the surface treatment throughout the device. Plasma-surface modification is one possible methodology for improving the biocompatible properties of biomaterials. To date, most studies

Manuscript received August 31, 2004; revised October 26, 2004.

J. L. Lauer and J. L. Shohet are with the Plasma Processing and Technology Laboratory and the Department of Electrical and Computer Engineering, the University of Wisconsin, Madison, WI 53706 USA.

R. M. Albrecht is with the Department of Animal Science, the University of Wisconsin, Madison, WI 53706 USA.

S. Esnault and J. S. Malter are with the Department of Pathology and Laboratory Medicine, the University of Wisconsin, Madison, WI 53706 USA.

U. H. von Andrian is with the Plasma Processing and Technology Laboratory and the Department of Electrical and Computer Engineering, the University of Wisconsin-Madison, Madison, WI 53706 USA. He is also with the Harvard Medical School, Boston, MA 02115 USA.

S. B. Shohet is with the Plasma Processing and Technology Laboratory and the Department of Electrical and Computer Engineering, the University of Wisconsin, Madison, WI 53706 USA. He is also with the University of California, San Francisco, CA 94143 USA.

Digital Object Identifier 10.1109/TPS.2005.844533 of plasma-modified biomaterials make use of a large-volume plasma reactor to modify the surface of a small disk or film substrate [1]-[4]. However, such reactors are not very practical for surface modification of small diameter tubing [5] and many small-scale biomedical devices [8]. This is because the plasma often does not penetrate along the entire length of the inside (lumenal surface) of the tubing, [6], [7] when the tubing is placed in a large-volume reactor, and thus only small portions near the ends of the tube are treated [5]. Also, complications in treating the side walls of narrow trenches and reservoirs of small-scale biomedical devices can cause further nonuniformities of the coating. This is because the flux of energetic plasma species to the bottom of trenches is often not the same as to the sidewalls.

Microplasma surface modification has the potential to treat surfaces of a variety of shapes and sizes that are contained in the small volume of a biomedical device. This could be done by embedding [8] microelectrodes into the structure of the device itself. With proper placement of the microelectrodes within the device, it may be possible to produce both bioadhesive and nonadhesive surface areas in the same device. This would allow for different surface-specific treatments of various chambers and channels throughout a device by connecting the leads of the electrodes to the appropriate excitation source (microwave [9]-[11], radiofrequency [12], [13], low frequency [14], [15], or direct current [16]-[18]) and inletting the proper [4] gas monomer(s) or surfactant coating. However, without a thorough understanding of the properties of such microplasmas, it is not likely that they can be controlled sufficiently to produce the desired surface modifications.

One of the simplest structures for a biomedical device is that of a tube. This includes, but is not limited to, artificial blood vessels (vascular grafts) of various diameters, as well as catheters. Plasma modification has been used to treat the lumenal surfaces of polyethylene terephthalate [19], [20] (PET, Dacron), expanded polytetrafluoroethylene, [19], [21] (ePTFE), polyethylene [22], [23] (PE), and polyurethane [7] tubes. The traditional method for coating the lumenal surfaces of these polymer tubes uses plasma polymerization (PP) of a monomer gas so as to modify the surface of the material directly or to introduce functional groups that can later be used to immobilize specific biomolecules or proteins. However, the key problem with previous approaches is the lack of the ability to control the uniformity of the coating along the length of the tubing. The uniformity includes that of the coating thickness, as well as its chemical composition [6]. This is due to the inherent drawback when 


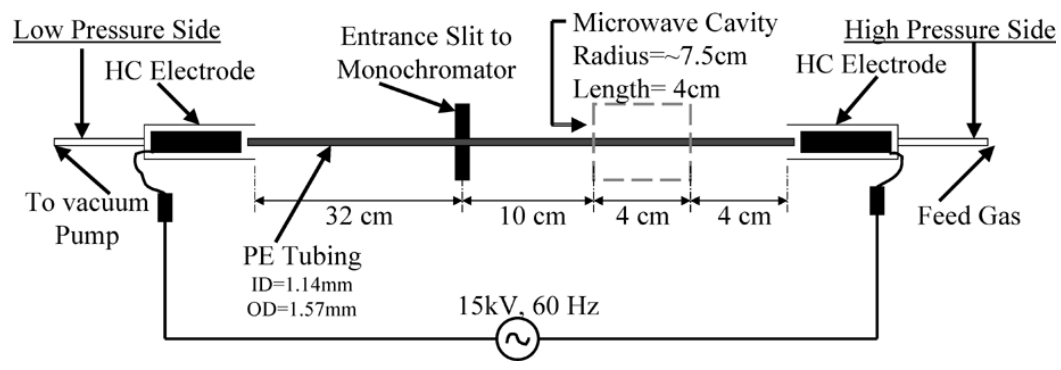

Fig. 1. Experimental configuration used to plasma-treat small-diameter PE tubing with the plasma diagnostics used to monitor the properties of the plasma discharge.

using PP where continuous consumption of the monomer gas and removal of the residual gases throughout the length of the tubing is a mandatory condition for the uniformity of the coating [5], [6].

In this paper, we describe a new approach for treating small-diameter polymer tubing [23] and we make use of two microplasma diagnostics to monitor the plasma properties during the treatment process. Hollow cathode (HC) electrodes [24]-[26] were placed in vacuum at each end of the PE tubing creating a microplasma along the entire length of the tubing. Instead of using PP, we use plasma immobilization (PI) [27]-[31], to immobilize a Poly(ethylene oxide) (PEO) surfactant [32], [33] to the lumenal surface of polyethylene (PE) tubing. We will show that immobilized PEO is useful for the reduction of adhering human platelets to the lumenal surface after exposure to blood flow in an in vitro blood circulation loop.

The benefits of using a microplasma discharge together with the PI technique will give us the ability to control uniformly the surface treatment of the lumenal surface along the entire length of small-diameter polymer tubes. In addition, the use of the appropriate diagnostics gives us the ability to examine the properties of microplasmas in general, as well as to find the optimal conditions for microplasma processing of biomaterials in a small volume.

\section{EXPERIMENTAL CONFIGURATION FOR MiCROPLASMA TREATMENT}

To prepare for PI, microplasma surface modification was used to modify the lumenal surface of small diameter PE tubing (I.D. $1.14 \mathrm{~mm}$, O.D. $1.57 \mathrm{~mm}$ ). A diagram of the plasma discharge configuration and the diagnostics used in this work is shown in Fig. 1. The surface modification was accomplished by placing $\mathrm{HC}$ electrodes at each end of the polymer tubing which was then evacuated at one end while feed gas was injected at the other end, as shown in Fig. 1. Two different HC electrode sizes were used at each end of the polymer tube. The low-pressure side had a $\mathrm{HC}$ electrode with an inner diameter of $8 \mathrm{~mm}$ and the high-pressure side $\mathrm{HC}$ electrode had a diameter of $6 \mathrm{~mm}$. The $\mathrm{HC}$ electrodes were contained in glass envelopes which were connected to each end of the polymer tube via a compressional fitting. It should be noted that the region outside of the radius of the polymer tube was at atmospheric pressure, thus the plasma was contained only inside of the tube. The electrodes where driven by a $15-\mathrm{kV}$ ac transformer, which was controlled with a
VARIAC. The corresponding discharge current through the tube was about $10 \mathrm{~mA}$.

Two plasma diagnostics were used to monitor the uniformity of the plasma along the length of the polymer tubing by measuring spectral components of the emitted light with a monochromator and the plasma electron density by measuring the shift in the resonant frequency of a microwave cavity. The tubing and the $\mathrm{HC}$ electrodes were attached to a movable table, so that regions of the tubing can be passed through the microwave cavity and in front of the entrance slit of the monochromator simultaneously. Thermocouple gauges monitored the pressure at each end of the PE tube. The pressure drop across the tube was controlled by pumping on both ends of the tubing and adjusting the throttle valves at each end of the tube, while inletting gas at one end. Variations in the properties of the treated tubing where observed when the plasma is not uniform along the length of the tubing. We will show that by controlling the pressure drop across the polymer tubing, the uniformity of the plasma treatment can be optimized. Long plasma exposure times had to be avoided to keep the PE tube from melting. To accomplish this, the PE tubes were exposed to a 1-s plasma pulse and then the tube was allowed to cool before the next pulse took place.

\section{Microplasma Diagnostics}

When a plasma is contained to a small volume or small crosssectional area, the plasma properties; such as electron density, chemical composition, temperature, spectral emission, etc., are often much different from that which is typically found in a conventional larger-volume reactor. Thus, the conditions under which microplasmas modify the surface of biomaterials will also be different. A thorough understanding of the properties of such plasmas is key to controlling the surface properties that can be obtained with microplasma discharges. This drives the necessity for noninvasive diagnostics, since immersing a probe into such plasmas is very likely to significantly perturb them from their normal operating conditions [34].

To determine the uniformity of the microplasma during the treatment of PE tubing, the microwave cavity diagnostic was used to obtain an estimate of the plasma density of the microplasma inside of a small-diameter polytetrafluoroethylene (PTFE) polymer tube. In addition, the intensity of the $3570 \AA$ Nitrogen line emitted from an air plasma discharge was monitored as a function of distance along the tube to estimate the variation of the gas composition along the tubing. 


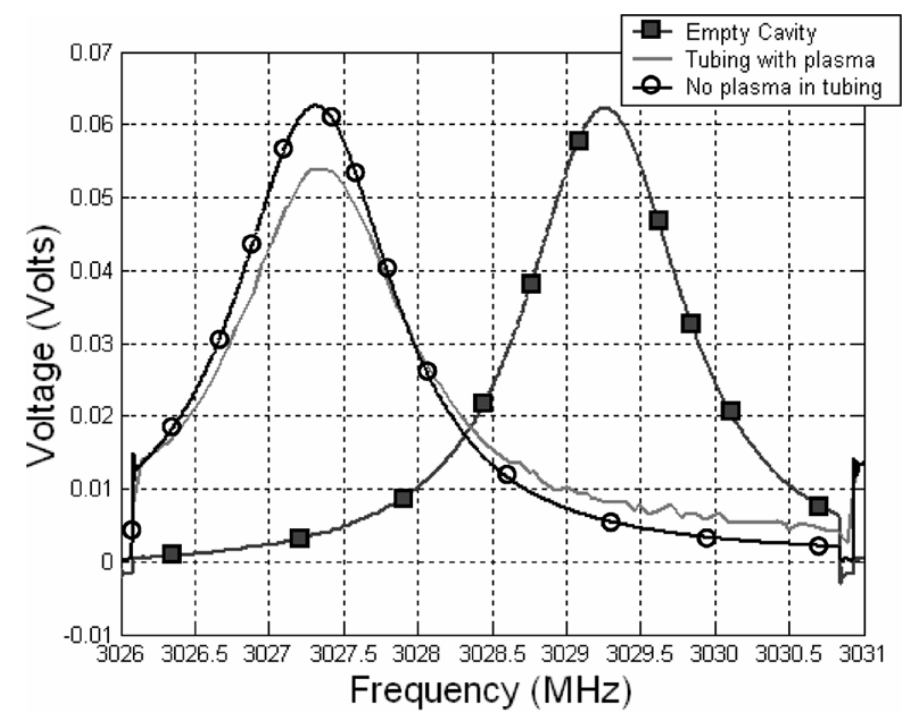

Fig. 2. $\mathrm{TM}_{010}$ mode as a function of frequency a) without the polymer tubing, b) with the polymer tubing, and c) with both the polymer tubing and plasma discharge present inside the microwave cavity.

\section{A. Microwave Cavity Measurements}

The microwave cavity method for measuring plasma density and collision frequency has been used for a long time [35]. However, with the emergence of microplasmas, the use of this diagnostic has been mostly overlooked. The microwave cavity method may be an ideal diagnostic for measuring the properties of such microplasmas, since a microwave cavity can be made into almost any arbitrary shape and a number of electromagnetic modes can be excited. Since the 1950s, right-circular cavities driven in the $\mathrm{TM}_{010}$ mode have been widely used in the microwave portion of the spectrum for plasma measurements [36], [37]. In this method, the plasma is placed along the axis of the cavity, and the change of resonant frequency and $Q$ value are measured [38]. From these measurements, the average plasma density [37], [39] and collision frequency [40] can be calculated.

For this work, a copper cylindrical microwave cavity of radius $75 \mathrm{~mm}$ and height $40 \mathrm{~mm}$ was constructed that had $\mathrm{aTM}_{010}$ resonant frequency of $3029 \mathrm{MHz}$. The output of a Hewlett-Packard 83623A synthesizer-sweeper was coupled through a half-turn small-diameter loop placed on the inner circular surface of the cavity half-way between the ends. An identical loop placed $180^{\circ}$ around the cavity from the input loop fed a diode detector. This arrangement can thus measure the transmission through the cavity as a function of frequency. Fig. 2 shows the results of three scans from 3026-3031 MHz. The three scans are as follows: 1) empty cavity; 2) empty PTFE tubing placed along the axis of the cavity; and 3) scan number 2 with an air plasma formed inside the tubing. Since PTFE tubing has a higher melting temperature than PE, it was used in the initial testing of the diagnostic. In Fig. 2, it can be seen that when no tubing is inside of the cavity, the resonant frequency of the $\mathrm{TM}_{010}$ mode is $3029.27 \mathrm{MHz}$. When the PTFE tubing (ID $=1.14 \mathrm{~mm}, \mathrm{OD}=1.57 \mathrm{~mm}$ ) was inserted along the axis of the microwave cavity, the resonant frequency of the $\mathrm{TM}_{010}$ mode shifted down by $2.03 \mathrm{MHz}$. This is because the dielectric constant of PE is greater than that of air and thus the

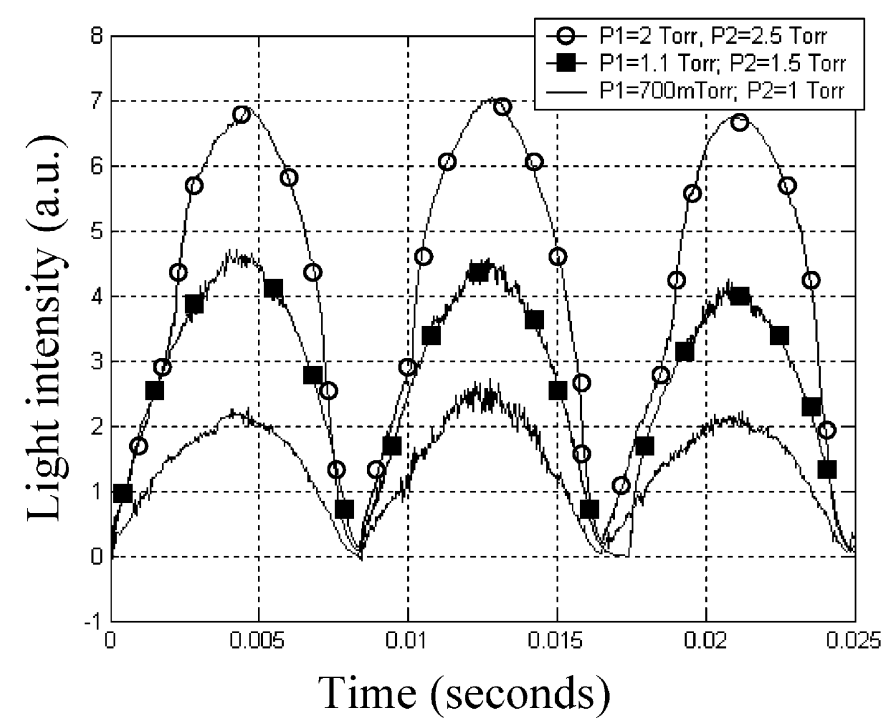

Fig. 3. Light emitted from the 3570- $\AA$ nitrogen line as a function of time over several ac periods for different feed pressures.

resonant frequency of the $\mathrm{TM}_{010}$ mode decreases [38]. When a plasma is created inside of the tubing, since the plasma's dielectric constant is less than that of vacuum, the resonant frequency of the $\mathrm{TM}_{010}$ mode shifts up and the $Q$ of the cavity gets smaller since the plasma is lossier than vacuum. This can be seen in Fig. 2 where both the upward shift and the increase in width of the resonance (lower $Q$ ) are evident. The shift of the resonant frequency caused by the presence of the plasma is small, but it is measurable and was found to be $0.03 \mathrm{MHz}$. Initial estimates of the plasma density were found using a previously developed model [39], which gave a value of about $4.8 \times 10^{10}$ electrons $/ \mathrm{cm}^{3}$ for the plasma electron density. During this measurement, the low-pressure side of the tubing was at 1.8 torr, the high-pressure side was at 2 torr and the microwave cavity was placed $20 \mathrm{~cm}$ from the high-pressure side of a 50-cm long PTFE tube.

\section{B. Monochromator Measurements}

Optical emission spectroscopy [41] provides both the identification and monitoring of light emitting species in the microplasma during operation. Fig. 3 shows the intensity of the emission from the $3570-\AA$ nitrogen line as a function of time over several alternating current periods, for three different air fill pressures. It can be seen that as the pressure is increased, the intensity of the light emitted from the plasma also increases. Thus, if a pressure drop is created along the length of a small-diameter polymer tube, the intensity of the light emitted from the microplasma as a function of distance along the tubing should provide information about the uniformity of the discharge.

To test this hypothesis, the intensity of light emitted from the plasma that passes through the wall of the PE tubing from the $3570-\AA$ line as a function of position along the length of a $50-\mathrm{cm}$ long polymer tubing was recorded. By changing the pressures at each end of the polymer tubing, we can control the total pressure drop across the tubing. Two conditions where chosen: 1) a uniform plasma case (low-pressure side $=140$ mtorr, high-pressure side $=190$ mtorr), and 2) a nonuniform 


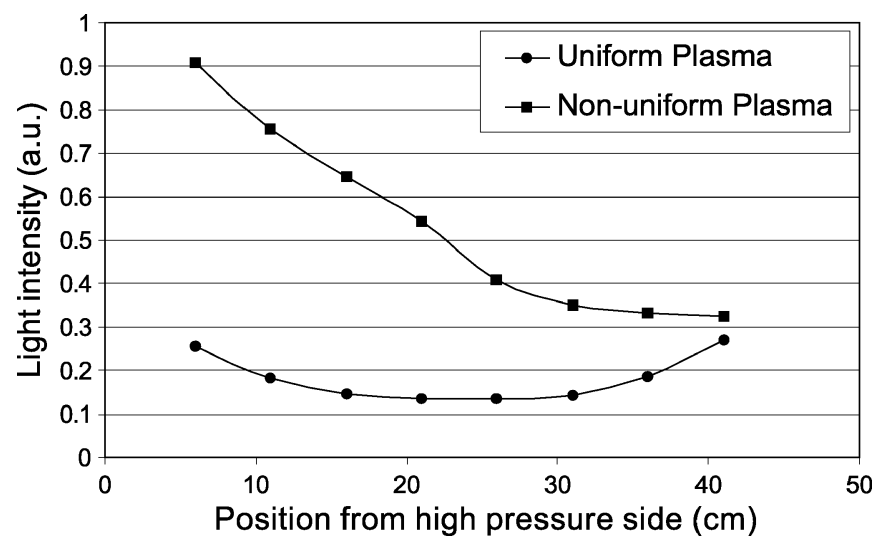

Fig. 4. Light emitted from the $3570-\AA$ nitrogen line as a function of position along the polymer tubing for two plasma conditions, uniform and nonuniform air-microplasma discharge conditions.

case (low-pressure side $=170$ mtorr, high-pressure side $=$ 550 mtorr). Thus, the pressure drop was about 50 mtorr for the uniform plasma and 380 mtorr for the nonuniform plasma. The intensity of the $3570-\AA$ line measured as a function of distance along the tubing, for the two cases is shown in Fig. 4. For the uniform plasma case, the light intensity reaches a minimum in the middle $20 \mathrm{~cm}$ of the tubing and increases at each end of the tubing. We believe that this is due to the fact that the $\mathrm{HC}$ electrodes are at each end of the tube, and thus the resulting electric fields and, therefore, the plasma is more intense at each end. However, when the pressure drop across the tubing is increased to 380 mtorr (nonuniform case), the light intensity measured along the tubing has a maximum at the high-pressure end and then it decays monotonically toward the low-pressure end of the tubing before reaching a constant level just before the $\mathrm{HC}$ electrode is reached. We conclude that the light emitted from the microplasma can become nonuniform along the tubing due to two factors: 1) if a large pressure drop is created across the tubing, and 2) proximity to the electrodes that create the plasma. It is important to determine whether the light intensity measured as a function of position along the length of the polymer tubing can be used to predict the uniformity of the surface treatment created by a uniform or nonuniform plasma discharge. To test this hypothesis, the treatment effectiveness along the lumenal surface of the tubing was evaluated using the capillary-rise method, which can be directly related to the contact angle.

\section{CAPILlARY-RisE METHOD}

Effectiveness of the plasma treatment on the lumenal surface of the PE tubing was evaluated using the capillary-rise (CR) method [7]. Use of the CR method allows us to monitor the uniformity of the contact angle inside the PE tubing as a function of distance along the tubing, which is believed to be very dependent on the lumenal-surface properties. The CR measurements of the PE tubing were obtained by cutting the $50-\mathrm{cm}$ long plasma-treated tubing into ten sections of 5-cm length. Each section was then dipped in a beaker of deionized water such that at least $4 \mathrm{~cm}$ of the section was submerged [7]. Then the tube was slowly withdrawn from the water, and the height of the water

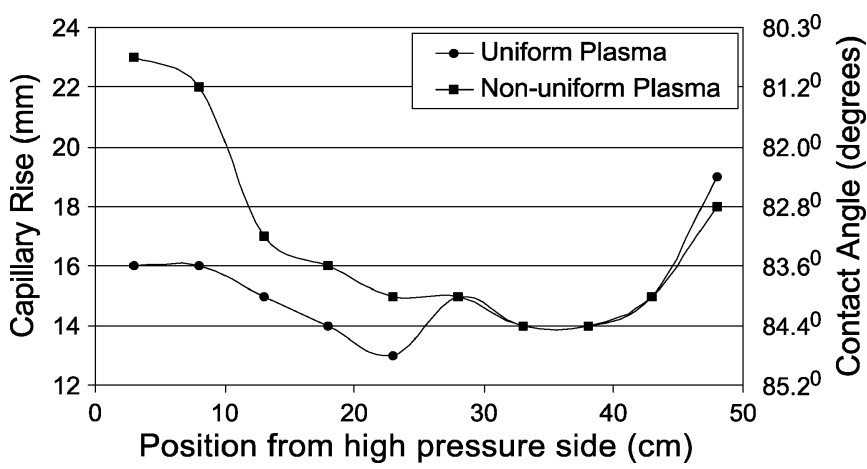

Fig. 5. Capillary rise measurements taken as a function of position along two PE tubes exposed to two different plasma conditions, a uniform and a nonuniform air-microplasma discharge.

column supported by the surface tension on the lumenal surface was measured. The contact angle of the lumenal surface is related to the height of the column of liquid by the expression which is obtained from the Young-Laplace equation [42]

$$
h=\frac{2 s \cos \theta}{n g r}
$$

where $h$ is the height of the liquid (it can be negative if the liquid falls below the surface of the water outside the tubing), $s$ is the surface tension of the liquid, $r$ is the radius of curvature of the tubing, $n$ is the density of the liquid, $g$ is the gravitational constant, and $\theta$ is the contact angle of the liquid on the capillary surface. The lumenal surface of the PE tubing (ID $=1.14 \mathrm{~mm}$, $\mathrm{OD}=1.57 \mathrm{~mm}$ ), in its original state from the vendor, exhibited a negative capillary rise of $6 \mathrm{~mm}$, which corresponds to a contact angle $\theta$ of approximately $92^{\circ}$, which is close to values that have been previously reported [31] for flat untreated PE surfaces. To determine whether a change in pressure drop along the tubing, during plasma treatment affects the CR measurements, the total pressure drop along the tubing was changed. The same plasma conditions used for the monochromator uniformity measurements were used here, which were: 1) uniform plasma (low-pressure side $=140$ mtorr, high-pressure side $=$ 190 mtorr), and 2) nonuniform plasma (low-pressure side $=$ 170 mtorr, high-pressure side $=550$ mtorr). For the uniform plasma case, the PE tube was exposed for two 1-s pulses, while for the nonuniform case, the tube was exposed for three 1-s pulses. An air plasma was used to treat the tubing in both cases. The results of the CR measurements, for the two cases, as a function of distance along the plasma treated tubing, is shown in Fig. 5. The left-hand scale shows the CR rise of the DI water and the corresponding contact angle is shown on the right-hand scale. It can be seen that for both cases, there is a minimum $\mathrm{CR}$ near the middle of the tubing. The CR increases near the ends of the tubing. Again, similarly to the monochromator measurements, we believe that this is due to the fact that the electrodes that create the plasma are located at the ends of the tubing, where the plasma is more intense. For the nonuniform case, the CR measured along the tubing has a maximum at the high-pressure end and then it drops toward the low-pressure side. This is very similar to the monochromator measurements under the same conditions. However, unlike the monochromator measurements, which flatten out at the low-pressure end, the CR keeps increasing toward the low-pressure side. We conclude that the 
CR measurements become more nonuniform along the tubing as a result of two factors: 1) when the pressure drop along the tubing is increased, and 2) the proximity to the electrodes.

Thus, we have shown that the intensity of light emitted from the microplasma as a function of position can be related to the uniformity of the plasma along the treated tubing. In addition, light intensity can be related to the CR along the tubing under similar plasma conditions. It is also important to note that for five or more 1-s pulses in an air-plasma, for both the uniform and nonuniform plasmas, the $\mathrm{CR}$ reaches a maximum of $27 \mathrm{~mm}(\theta \sim$ $79^{\circ}$ contact angle), which is uniform along the whole length of the PE tubing. Therefore, uniformity of plasma treatment can be measured with the CR method alone. However, it is expected that without careful optimization of the plasma, the ends of the tubing where the plasma is more intense may be degraded before the lumenal surface near the middle of the tube is fully treated. This may become more of a problem as the length of the tube is increased.

\section{COATING AND IMMOBILIZATION OF PEO SURFACTANTS ONTO PE TUBING}

The PI technique consists of a two-step process [43]. First, a surfactant is coated on the surface to be modified and then it is exposed to an inert gas plasma. During the inert-gas plasma exposure, crosslinking between the surfactant and the polymer substrate may occur, thus covalently coupling part of the precoated surfactant to the lumenal surface of the tubing by the Crosslinking by Active Species of INert Gases ("CASING") method [30]-[32]. Since an inert gas is used, there is no significant change in gas-phase plasma chemistry along the tubing, since argon is not consumed by the surfactant or the substrate during plasma exposure [32]. Thus, this technique is ideal for treating the lumenal surface of small-diameter polymer tubing, since the consumption of gas is negligible, making it easier to control the uniformity of the microplasma treatment.

To improve wetting of the PE surface by a $10 \% \mathrm{w} / \mathrm{v}$ aqueous PEO surfactant (Brij 98) solution, the hydrophilicity of the lumenal surface of the PE tubing was increased. This was accomplished by introducing polar functional groups at the surface by means of plasma treatment [43]. The inside of the PE tubing was exposed to four 1-s pulses of a uniform air-plasma. The tubing was then removed from the reactor, and the PEO surfactant was injected into the PE tubing and remained in contact with the lumenal surface for $30 \mathrm{~min}$ at room temperature (RT). Subsequently, the surfactant was withdrawn, and the tube was air dried at RT for 30 min while the solvent evaporated, leaving behind a thin coating of PEO polymer on the lumenal surface. The precoated tube was then reconnected to the vacuum system and exposed to an argon plasma, under uniform-plasma conditions, for a total of 101 -s pulses. After the final plasma treatment, the tube was flushed with DI water for 5 min and then soaked in fresh DI water before blood compatibility testing was undertaken.

\section{Blood-Copatibility Testing}

To determine whether PEO that has been plasma-immobilized on the lumenal surface of PE tubing can reduce the

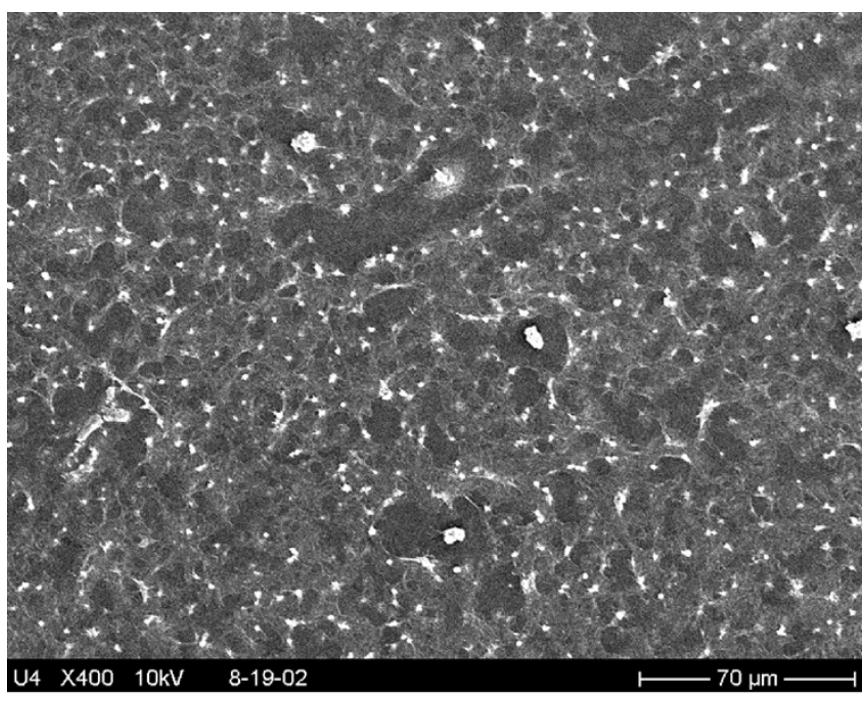

(a)

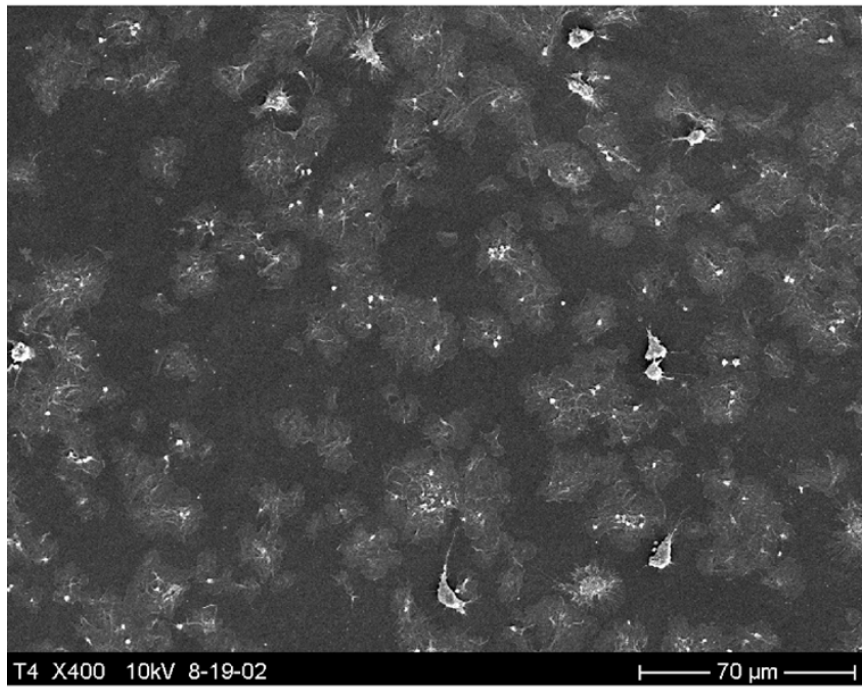

(b)

Fig. 6. SEM images of the lumenal surface of untreated and PEO treated PE tubing after exposure to blood flow at a rate of $0.75 \mathrm{ml} / \mathrm{min}$ at $37{ }^{\circ} \mathrm{C}$ for $1 \mathrm{~h}$.

adhesion of human blood platelets, an in vitro blood-circulation loop was constructed. Detailed procedures of the protocol for the blood compatibility testing of the plasma modified tubes have been described elsewhere [23] and are only briefly described here. A peristaltic pump circulated blood through two separate 26-cm long PE tubes, one of which was PEO plasma-immobilized, while the other remained untreated. Initially, the blood-flow direction passed through the treated tubing, then through the peristaltic pump, and finally through the untreated tubing before returning to the reservoir. After $30 \mathrm{~min}$, the flow direction was reversed. After blood flow, the remaining cells that were attached to the lumenal surface of the tubes were fixed and dehydrated, and then prepared for scanning electron microscopy (SEM) imaging. Figs. 6 and 7 show SEM images of both the untreated and PEO-treated tubes after blood exposure. To facilitate comparison, and to utilize the uniformly treated region of the tubing, SEM images were 


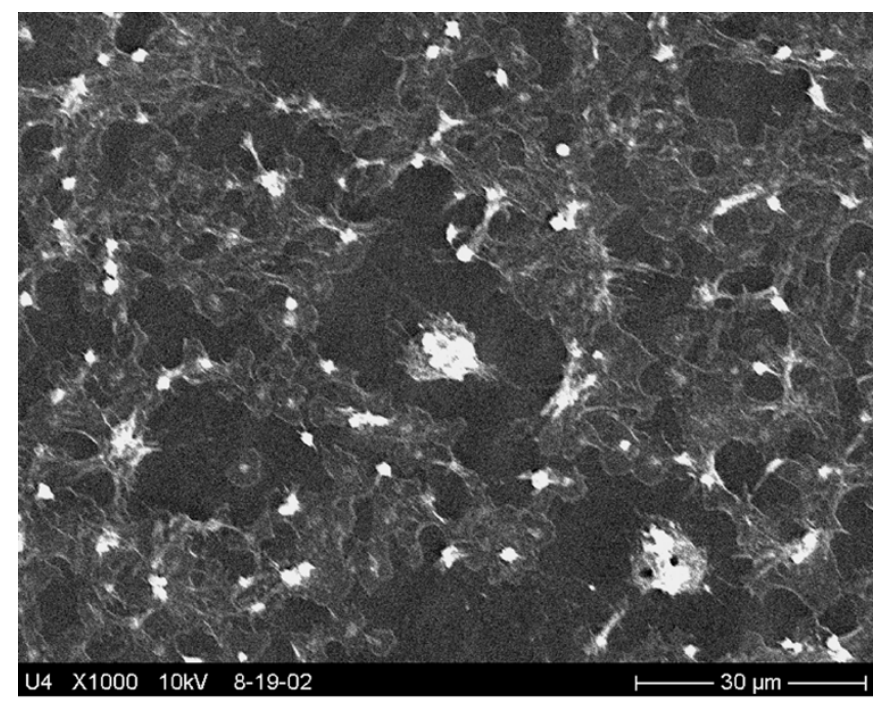

(a)

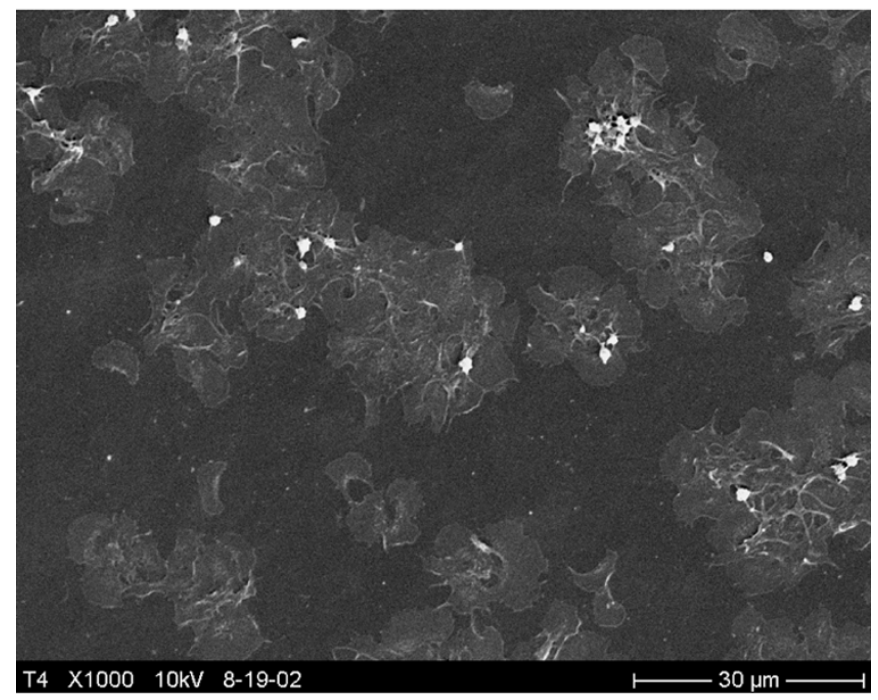

(b)

Fig. 7. SEM images of the lumenal surface of untreated and PEO treated PE tubing after exposure to blood flow under higher magnification than Fig. 8.

obtained at the same point, near the middle of both the treated and untreatd tubes.

Fig. 6(a) and (b) shows the adhering blood components (mostly platelets) on the lumenal surface of the untreated and PEO-treated tubes, respectivly. It can be seen that the number of platelets adhering to the PEO-treated tubing is much less than the number adhering to the untreated PE tube. In Fig. 6(a), it can be seen that numerous microthrombi have formed on the lumenal surface of the untreated PE tube after one hour of exposure to heparinized blood. In Fig. 6(b), it can be seen that there are a relatively few number of spreading platelets on the PEO-treated tube, and even fewer bases for microthrombi. Under higher magnification, shown in Fig. 7(a) and 7(b), it can be seen that the fibrin network is far more complex on the untreated tubing compared to the treated tubing. Thus, we conclude that the number of adhering platelets on the lumenal surface of PE tubing can be reduced by immobilizing a PEO surfactant to the lumenal surface of the tubing using a microplasma discharge.

\section{SUMMARY}

A microplasma was created with $\mathrm{HC}$ electrodes which are placed at each end of the tube. This discharge configuration has many advantages compared to previous techniques. They are as follows: 1) it is an inexpensive method to create a microplasma inside small-diameter polymer tubing, and 2) when the plasma is created, it extends along the entire length of tubing thus eliminating the need for movable electrodes or tubing.

Two microplasma diagnostics were used to monitor the plasma properties. It was shown that by suitably controlling the pressure drop along the tube, the degree of plasma uniformity as a function of distance along the tubing can be optimized. Emitted light from the plasma was fed into a monochromator at various positions along the PE tube to measure the uniformity. The monochromator measurements were then related to the capillary rise measured along the length of the polymer tubing for uniform and nonuniform plasma conditions. It was shown that a nonuniform plasma treatment can be due to two factors: 1) a pressure drop created across the PE tubing, and 2) proximity to the electrodes. A microwave-cavity diagnostic was used to measure the density of an air-microplasma-a value of $4.8 \times 10^{10}$ electrons $/ \mathrm{cm}^{3}$ was obtained.

PI was used to immobilize a PEO surfactant to the lumenal surface of small-diameter PE tubing. It was seen that the use of this method to immobilize PEO surfactants to the lumenal surface of plasma-treated PE tubing showed a significant decrease in the number of platelets adhering to the lumenal surface compared with untreated PE tubing.

\section{REFERENCES}

[1] M. Sheu, M. S. Wagner, D. G. Castner, B. D. Ratner, and T. A. Horbett, "Multivariate surface analysis of plasma-deposited tetraglyme for reduction of protein adsorption and monocyte adhesion," Langmuir, vol. 19, pp. 1692-1699, 2003.

[2] M. Sheu, L. Martinson, M. S. Wagner, D. G. Castner, B. D. Ratner, and T. A. Horbett, "PEO-like plasma polymerized tetraglyme surface interactions with leukocytes and proteins: In vitro and in vivo studies," $J$. Biomater. Sci. Polymer Edn., vol. 13, pp. 367-390, 2002.

[3] M. Sheu, Y. V. Pan, M. S. Wagner, K. D. Hauch, D. G. Castner, B. D. Ratner, and T. A. Horbett, "Inhibition of monocyte adhesion and fibrinogen adsorption on glow discharge plasma deposited tetraethlyene glycol dimethyl ether," J. Biomater. Sci. Polymer Edn., vol. 12, pp. 961-978, 2001.

[4] S. Dumitriu, Ed., Polymeric Biomaterial. New York: Marcel Dekker, 2001.

[5] Y. Matsuzawa and H. Yasuda, "Semi-continuous plasma polymerization coating applied onto the inside surface of plastic tubing," Org. Coat. Appl. Polym. Sci. Proc., vol. 47, p. 397, 1982.

[6] - "Semicontinuous plasma polypolymerization coating onto the inside surface of plastic tubing," J. Appl. Poly. Sci. Appl. Poly. Sym., vol. 38, p. 65, 1984.

[7] P. V. Narayanan, "Surface functionalization by RF plasma treatment of polymers for immobilization of bioactive-molecules," J. Biomater. Sci. Polymer Edn., vol. 6, pp. 181-193, 1994.

[8] T. Ichiki, Y. Sugiyama, R. Taura, T. Koidesawa, and Y. Horiike, "Plasma applications for biochip technology," Thin Solid Films, vol. 435, pp. 62-68, 2003.

[9] F. Iza and J. A. Hopwood, "Low-power microwave plasma source based on a microstrip split-ring resonator," IEEE Trans. Plasma Sci., vol. 31, no. 4, pp. 782-787, Aug., 2003. 
[10] A. M. Bilgic, U. Engel, E. Voges, M. Kuckelheim, and J. A. C. Broekaert, "A new low-power microwave plasma source using microstrip technology for atomic emission spectrometry," Plasma Sources Sci. Technol., vol. 9, pp. 1-4, 2000.

[11] A. M. Bilgic, E. Voges, U. Engel, and J. A. C. Broekaert, "A low-power $2.45 \mathrm{GHz}$ microwave induced helium plasma source at atmospheric pressure based on microstrip technology," J. Anal. At. Spectrom., vol. 15, pp. 579-580, 2000.

[12] Y. B. Guo and F. C. N. Hong, "Radio-frequency microdischarge arrays for large-area cold atmospheric plasma generation," Appl. Phys. Lett., vol. 82, pp. 337-339, 2003.

[13] H. Yoshiki, A. Oki, H. Ogawa, and Y. Horiike, "Generation of a capacitively coupled microplasma and its application to the inner-wall modification of a poly(ethylene terephthalate) capillary," J. Vac. Sci. Technol. A Vac. Surf. Films, vol. 20, pp. 24-29, 2002.

[14] N. Gherardi, G. Gouda, E. Gat, A. Ricard, and F. Massines, "Transition from glow silent discharge to micro-discharges in nitrogen gas," Plasma Sources Sci. Technol., vol. 9, pp. 340-346, 2000.

[15] J. A. Hopwood, "A microfabricated inductively coupled plasma generator," J. Microelectromech. Syst., vol. 9, pp. 309-313, 2000.

[16] C. G. Wilson, Y. B. Gianchandani, R. R. Arslanbekov, V. Kolobov, and A. E. Wendt, "Profiling and modeling of dc nitrogen microplasmas," $J$. Appl. Phys., vol. 94, pp. 2845-2851, 2003.

[17] T. Ono, D. Y. Sim, and M. Esashi, "Micro-discharge and electric breakdown in a micro-gap," J. Micromech. Microeng., vol. 10, pp. 445-451, 2000.

[18] L. D. Biborosch, O. Bilwatsch, S. Ish-Shalom, E. Dewald, U. Ernst, and K. Frank, "Microdischarges with plane cathodes," Appl. Phys. Lett., vol. 75, pp. 3926-3928, 1999.

[19] T. Chandy, G. S. Das, R. F. Wilson, and G. H. R. Rao, "Use of plasma glow for surface-engineering biomolecules to enhance bloodcompatibility of Dacron and PTFE vascular prosthesis," Biomaterials, vol. 21, pp. 699-712, 2000.

[20] D. Kiaei, A. S. Hoffman, B. D. Ratner, and T. A. Horbett, "Interaction of blood with gas discharge treated vascular grafts," J. Appl. Polmer. Sci: Appl. Polymer Sym., vol. 42, p. 269, 1988.

[21] D. Y. Tseng and E. R. Edelman, "Effects of amide and amine plasmatreated ePTFE vascular grafts on endothelial cell lining in an artificial circulatory system,” J. Biomed. Mater. Res., vol. 42, pp. 188-198, 1998.

[22] J.-C. Lin and S. L. Cooper, "Surface characterization and ex vivo blood compatibility study of plasma-modified small diameter tubing: Effect of sulphur dioxide and hexamethyl-disiloxane plasmas," Biomaterials, vol. 16, pp. 1017-1023, 1995.

[23] J. L. Lauer, J. L. Shohet, R. M. Albrecht, C. Pratoomtong, R. Murugesan, S. Esnault, J. S. Malter, U. H. von Andrian, R. D. Bathke, and S. B. Shohet, "Reduced adhesion of human blood platelets to polyethylene tubing by microplasma surface modification," J. Appl. Phys., vol. 96, pp. 4539-4546, 2004.

[24] K. H. Schoenbach, M. Moselhy, W. Shi, and R. Bentley, "Microhollow cathode discharges," J. Vac. Sci. Technol. A, Vac. Surf. Films, vol. 21, pp. 1260-1265, 2003.

[25] K. H. Schoenbach, R. Verhappen, T. Tessnow, F. E. Peterkin, and W. W. Byszewski, "Microhollow cathode discharges," Appl. Phys. Lett., vol. 68, pp. 13-15, 1996.

[26] S. J. Park and J. G. Eden, "13-30 micron diameter microdischarge devices: Atomic ion and molecular emission at above atmospheric pressures," Appl. Phys. Lett., vol. 81, p. 4127, 2002.

[27] J. P. Lens, J. G. Terlingen, G. H. Engbers, and J. Feijen, "Preparation of heparin-like surfaces by introducing sulfate and carboxylate groups on poly(ethylene) using an argon plasma treatment," J. Biomater. Sci. Polymer Edn., vol. 9, pp. 357-373, 1998.

[28] — , "Introduction of sulfate groups on poly(ethylene) surfaces by argon plasma immobilization of sodium alkyl sulfates," Polymer, vol. 39, pp. 3437-3444, 1998.

[29] — , "Introduction of carboxylate groups at poly(ethylene) surfaces by argon plasma immobilization of sodium salts of fatty acids," Langmuir, vol. 13, pp. 7052-7062, 1997.

[30] M. S. Sheu, A. S. Hoffman, and J. Feijen, "A glow discharge treatment to immobilize poly(ethylene oxide)/poly(propylene oxide) surfactants for wettable and nonfouling biomaterials," J. Adhesion Sci. Technol., vol. 6, pp. 995-1009, 1992.

[31] M. S. Sheu, A. S. Hoffman, J. G. A. Terlingen, and J. Feijen, "A new gas discharge process for preparation of nonfouling surfaces on biomaterials," Clinical Materials, vol. 13, p. 41, 1993.
[32] M. S. Sheu, A. S. Hoffman, B. D. Ratner, J. Feijen, and J. M. Harris, "Immobilization of polyethylene oxide surfactants for nonfouling biomaterial surfaces using an argon glow discharge treatment," J. Adhesion Sci. Technol., vol. 7, pp. 1065-1076, 1993.

[33] J. P. Lens, P. H. Harmsen, E. M. Ter Schegget, J. A. Terlingen, G. H. Engbers, and J. Feijen, "Immobilization of fuctionalized alkyl-poly(ethylene oxide) surfactants on poly(ethylene) surfaces by means of an argon plasma treatment," J. Biomater. Sci. Polymer. Edn., vol. 8, pp. 963-982, 1997.

[34] O. B. Minayeva and J. Hopwood, "Langmuir probe diagnostics of a microfabricated inductively coupled plasma on a chip," J. Appl. Phys., vol. 94, pp. 2821-2828, 2003.

[35] B. Agdur and B. Enander, "Resonances of a microwave cavity partially filled with a plasma," J. Appl. Phys., vol. 33, pp. 575-581, 1962.

[36] K. B. Persson, "Limitations of the microwave cavity method of measuring electron densities in a plasma," Phys. Rev., vol. 106, pp. 191-195, 1957.

[37] K. I. Thomassen, "Microwave plasma density measurements," J. Appl. Phys., vol. 36, pp. 3642-3644, 1965.

[38] S. Li, C. Akyel, and R. G. Bosisio, "Precise calulations and measurements on the complex dielectric constant of lossy materials using $\mathrm{TM}_{010}$ cavity perturbation techniques," IEEE Trans. Microwave Theory Tech., vol. MTT-29, pp. 1041-1046, 1981.

[39] J. L. Shohet and A. J. Hatch, "Eigenvalues of a microwave cavity filled with a plasma of variable radial density," J. Appl. Phys., vol. 41, pp. 2610-2618, 1970.

[40] L. Gould and S. C. Brown, "Methods of measuring the properites of ionized gases at high frequencies. IV. A null method of measuring the discharge admittance," J. Appl. Phys., vol. 24, pp. 1053-1056, 1953.

[41] R. d'Agostino, Ed., Plasma Deposition, Treatment, and Etching of Polymers. New York: Academic, 1990.

[42] A. W. Adamson and A. P. Gast, Physical Chemistry of Surfaces. New York: Wiley, 1997.

[43] J. P. Lens, J. G. Terlingen, G. H. M. Engbers, and J. Feijen, "Coating of anionic surfactants onto poly(ethylene) surfaces studied with X-ray photoelectron spectroscopy," Langmuir, vol. 14, pp. 3214-3222, 1998.

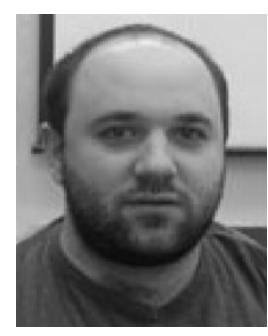

Jason L. Lauer (S'00-M'04) received the B.S. and M.S. degrees in electrical engineering, in 2001 and 2003, respectively, from the University of Wisconsin, Madison, where he is currently working toward the Ph.D. degree in the plasma processing and technology laboratory.

He spent the summer of 2004 at the Naval Research Laboratory, Washington, DC, working in the area of vacuum ultraviolet radiation.

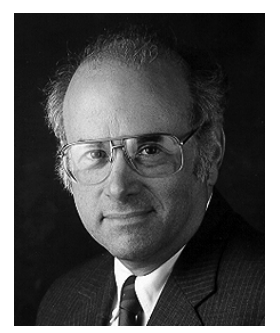

J. Leon Shohet (S'56-M'62-SM'72-F'78) received the $\mathrm{Ph} . \mathrm{D}$. degree in electrical engineering from Carnegie Mellon University, Pittsburgh, PA, in 1961.

He served on the faculty of Johns Hopkins University, Baltimore, MD, before joining the University of Wisconsin faculty in 1966 and was appointed Professor of Electrical and Computer Engineering in 1971. He is the Director of the Plasma Processing and Technology Laboratory and is the Founding Director of the University's NSF Engineering Research Center for Plasma-Aided Manufacturing, as well as the past Chairman of the Department of Electrical and Computer Engineering. $\mathrm{He}$ is the author of two textbooks on plasma science, over 150 journal articles, and more than 440 conference papers. He holds eight patents. His research interests include plasma-aided manufacturing, fusion, (especially waves, instabilities, heating, confinement and diagnostics), communications, magnetohydrodynamics, electromagnetic field theory, biophysics, and nonlinear science.

Dr. Shohet is a Fellow of the American Physical Society. He received the Frederick Emmons Terman award of the American Society for Engineering Education, the Merit Award of the IEEE Nuclear and Plasma Sciences Society, the IEEE Richard F. Shea Award, the IEEE Plasma Science Prize, the IEEE Centennial Medal, and the John Yarwood Memorial Medal from the British Vacuum Council. He founded the IEEE TRANSACtions On Plasma ScIENCE in 1973. 


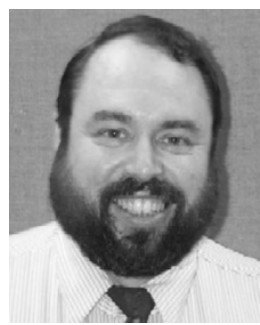

Ralph M. Albrecht received the B.S. degree in microbiology and the Ph.D. degree in microbiology and immunology from the University of Wisconsin, Madison, in 1967 and 1976, respectively.

From 1976 to 1985 , he held a joint appointment in the Department of Pediatrics in the School of Medicine and the Department of Pharmacology of the School of Pharmacy at the University of Wisconsin, Madison, where he held the rank of Associate Professor. Since 1985, he has held a joint appointment in the College of Agriculture and Life Sciences, Department of Animal Sciences; School of Pharmacy, Department of Pharmacology; and School of Medicine, Department of Pediatrics where he currently holds the rank of Professor. He serves as the Director of the Biological and Biomaterials Preparation, Imaging, and Characterization Laboratory at the University of Wisconsin, Madison.

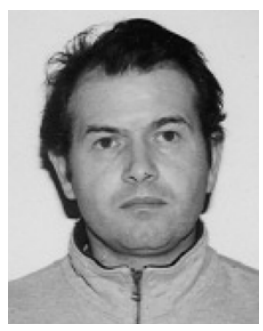

Stephane Esnault received the Maitrise degree in cellular biology from the Rennes I University, Rennes, France, in 1989, the DEA degree in microbiology from the Pharmacy University, Paris, France, in 1992, and the Ph.D. degree in immunology from the Pharmacy University, Reims, France, in 1996.

From 1997 to 2002, he was a Postdoctoral Fellow in the Department of Pathology, the University of Wisconsin, Madison and has been an Assistant Scientist in the Department of Pathology, the University of Wisconsin-Madison since 2002. His research interests include gene regulation in human blood cells

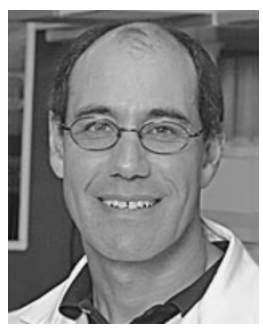

James S. Malter received the A.B. degree from Dartmouth College, Dartmouth, NH, in 1978 and the M.D. degree from Washington University, St. Louis, MO, 1983.

He has been on the faculty of the University of Wisconsin, Madison, School of Medicine, since 1988, where he currently holds the rank of Professor of Pathology.

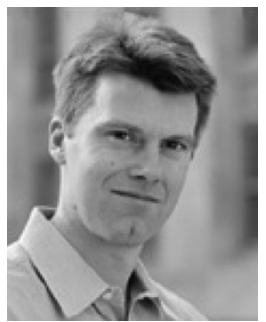

Ulrich H. von Andrian received the M.D. degree and the Ph.D. in neurology and neurosurgery from Ludwig-Maximilians University, Munioh, Germany, in 1989 and 1992, respectively.

He was a Postdoctoral Fellow at the LaJolla Institute for Experimental Medicine, as well as a visiting Scholar in the Department of Applied Mechanics and Engineering Sciences, University of California, San Diego from 1989 to 1991, a Postdoctoral Fellow in the Laboratory of Immunology and Vascular Biology in the Department of Pathology, Stanford Medical Center, Stanford, CA, from 1991 to 1993, and has been on the faculty of Harvard Medical School and the Center for Blood Research, Boston, MA, since 1994, where he holds the rank of Associate Professor of Pathology.

Dr. von Andrian is a Member of the European Academy of Sciences and the American Society for Cell Biology. In 2004, he was awarded the Amgen Outstanding Investigator Award.

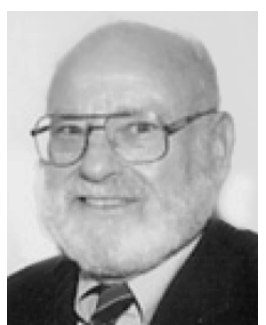

Stephen B. Shohet received the A.B. degree from Harvard College, Boston, MA, in 1956 and the M.D. degree from Harvard Medical School, Boston, MA, in 1960 .

From 1962 to 1965 , he was with the National Cancer Institute. From 1966 to 1968, he was on the staff of the Peter Bent Brigham Hospital, Boston, MA. From 1969 to 1971, he was Assistant Professor of Pediatrics at Children's Hospital, Harvard Medical School. Since 1971, he has been on the faculty of the Unviversity of California, San Francisco Medical Center, where he holds the dual appointments of Emeritus Professor of Laboratory Medicine and Professor of Medicine. His areas of interest include hematology, leukemias, lymphomas, white-cell diseases, blood clotting, thrombosis, bleeding, anemias, red-cell disorders, platelet problems, and the hematologic consequences of toxic exposure. 